\title{
Mobility Prediction using Modified RBF Network
}

\author{
Mohammad F. Hassanin \\ Computer Science Department, Faculty of \\ Computers and information, Fayoum University, \\ Egypt
}

\author{
Amr Badr \\ Computer Science Department, Faculty of \\ Computers and information, Cairo University, Egypt
}

\begin{abstract}
Recently, with the wide increase use of mobile devices, users are using it to achieve different types of tasks like multimedia and internet services. Many studies have been introduced to identify user's behaviors. The most common factor is the location. By knowing user's locations, set of required tasks can be provided for user. Mobility prediction is to predict the user's next location while moving. The recent advances in mobile phones like Global Positioning System (GPS) make it much easier to achieve such tasks. In this paper, modified Radial Basis Function Networks (RBF Network) algorithm is developed to predict user's locations. Proposed approach depend on clustering data using DBCLUM then using backpropagation algorithm for classifying data in on time using RBF Network. Experiment applied using weka 3.6 on Geolife dataset. Finally, prediction accuracy reported as $90 \%$.
\end{abstract}

\section{Keywords}

Mobility Prediction, Neural Network, GPS, Location Based Service.

\section{INTRODUCTION}

Mobility Prediction of wireless devices gives the user the availability for continuous services access like internet services and social media websites. Network operators must save the demanded services for user anywhere and anytime. So the location is the most greatest common factor to resolve this issue. Location-based services are evolving recently including finding locations like nearest hospital, nearest restaurants or visitor guide and so on. Other services concentrated on understanding and identifying user's behaviors. Hence, provide user with required services based on his attitude. Many researchers have studied this problem as in $[1,2]$ to detect significant locations and predict user movement as well as detect user behavior. Others focused on detecting transportation mode whether walking, driving a car or passenger in train. Hence, predicting user movement easily as [3] showed. To predict user location or behavior, movement pattern must be built first. Building human movement pattern is an area of interest for context-aware services [4, 5], Vehicle routing [6], and Mobility prediction and multicasting in wireless networks [7].

Radial basis function networks have two distinct layers as shown in figure 1. The bottom layer consists of a set of basis functions while each one represented as a function of the distance between two patterns; the input and the prototype. Generally, standard basis function is the Gaussian:

$$
\emptyset_{\mathrm{j}}=\exp \left(-\frac{\sum_{\mathrm{i}=1}^{\mathrm{n}}\left(\mathrm{x}_{\mathrm{j}}-\mathrm{w}_{\mathrm{ij}}\right)^{2}}{2 \sigma^{2}}\right)
$$

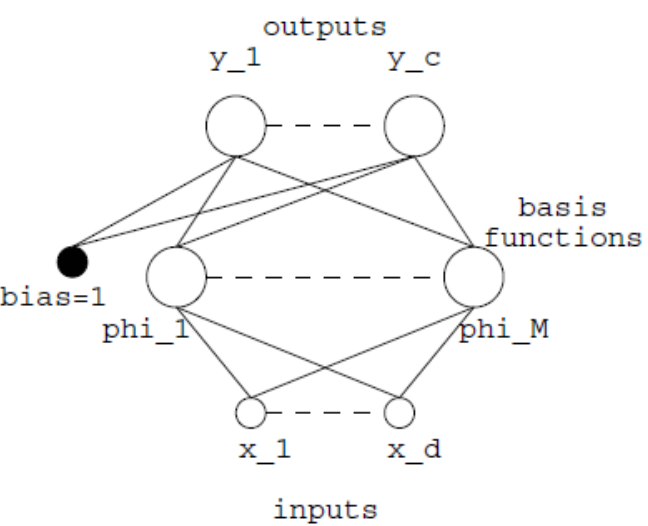

Fig 1: generic RBF Network

The top layer is responsible for outputting the weighted sum of basis functions. Equation for single output is shown in equation 2 .

$\mathrm{y}_{\mathrm{k}}(\mathrm{x})=\sum_{\mathrm{j}=1}^{\mathrm{M}} \mathrm{W}_{\mathrm{kj}} \emptyset_{\mathrm{j}}(\mathrm{x})+\mathrm{W}_{\mathrm{k}, \mathrm{bias}}$

The paper is organized as follows. The next section describes related works pertaining mobility prediction in the context of mobile computing. Next, we describe our prediction framework. Subsequently, results presented on predicting next user location.

\section{RELATED WORKS}

Mobility prediction has been handled and tested by many algorithms. In [8], used mobility motion prediction to predict data and recorded results were $95 \%$ of efficiency. In [9], a probabilistic model is used with Dempster-Shafer theory of evidential reasoning. The system collects evidence for required location and predicts the trajectory.

Statistical methods or state space methods like markov and hidden markov model (HMM) are used to predict the next location. In $[10,11]$ studies showed that markov is better than HMM in mobility prediction. Extensions for these methods also introduced like mobility markov chain to handle higher order of states corresponded to previously visited locations and results recorded are $95 \%$.

Data mining methods also has been used to predict next locations. In [12], used association rules to extract user's patterns and match with database to select best association rule. Recorded results were .86 as precision and .82 as recall. In [13], three-phase algorithm is developed firstly, mine user patterns, extract mobility patterns and finally accomplish mobility prediction. Others used neural networks algorithms to predict next user's locations as in $[14,15]$.

Hybrid techniques also have been tested for mobility prediction. In [16], used information extracted from movement pattern together with motion functions. In [17] 
used geographic and semantic features based on clustering while it assembles similar locations together.

\section{THE PROPOSED ALGORITHM}

RBF Network algorithm is modified to train and learn movement patterns. The key idea is to use Density-based clustering to detect the real data structure because we deal with GPS data. Clustering process produces both the number of nodes and the respective kernel parameters. The initial estimation of the connecting weights is the second step to optimize the parameters. All the above parameter values are fed into the back-propagation method to proceed with classification step. Figure 2 shows the proposed RBF Network algorithm used for mobility prediction and the phases of learning as follows:

\section{A. Clustering using DBCLUM}

Density-Based Clustering and Merging (DBCLUM) [18] is used as a clustering algorithm. We used DBCLUM which is a density-based clustering algorithm because it can cluster data without knowin a predefined number of clusters and can cluster with arbitrary shapes.

\section{B. Labeling Dataset}

To classify unsupervised data, labeling data is a must. Cluster label is considered as a class value.

\section{Remove noise}

Because DBCLUM is able to detect noise data and these noisy points halt the classification process, noisy data must be eliminated before going on learning phase.

\section{Initializing Parameters for Radial Basis Functions}

Gaussian basis functions, as in equation 1, each have two parameters $\mu \mathrm{j}$ and $\sigma \mathrm{j}$, for the $\mathrm{jth}$ the basis function $\emptyset \mathrm{j}$. Finding these parameters determined through density-based clustering phase.

$\mu_{\mathrm{j}}=\frac{1}{\mathrm{~N}} \sum_{\mathrm{n}=1}^{\mathrm{N}} \mathrm{x}^{\mathrm{n}}$

$\sigma_{\mathrm{j}}=\sqrt{\frac{1}{\mathrm{~N}} \sum_{\mathrm{n}=1}^{\mathrm{N}}\left(\mathrm{x}^{\mathrm{n}}-\mu_{\mathrm{j}}\right)^{\mathrm{T}}\left(\mathrm{x}^{\mathrm{n}}-\mu_{\mathrm{j}}\right)}$

Finally, weight matrix is computed by the typical method of Backpropagation Algorithm.

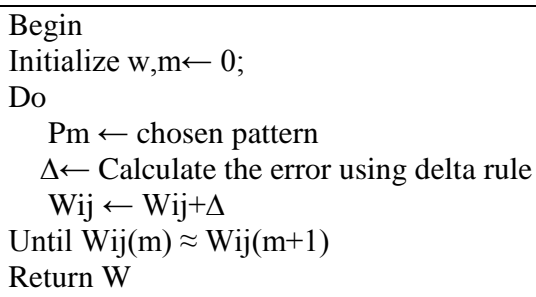

\section{E. Back Propagation Learning}

Back Propagation Learning Algorithm is a one of most popular supervised learning techniques because it is powerful, useful and many training methods are considered as a modification of it. Proposed method uses number of hidden unites which is equal to number of clusters formed by DBCLUM algorithm. The initial parameter estimations of $\sigma$, and which were calculated in the previous step are fed into the back-propagation.

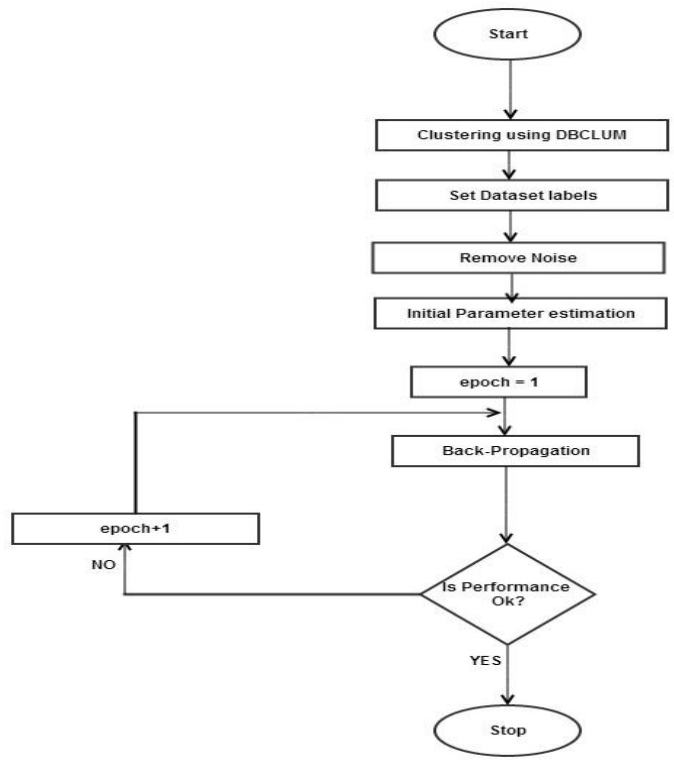

Fig 2: modified RBF Network

\section{EXPERIMENTAL RESULTS}

The experimental results are carried out on Geolife Trajectories 1.3 dataset [19]. Geolife is a GPS trajectory dataset by 178 users. Every record in dataset has seven fields as table 1 shows.

Table 1: Example of reocord of GeoLife

\begin{tabular}{|c|c|}
\hline Latitude & 39.906631 \\
\hline Longitude & 116.385564 \\
\hline All & 0 \\
\hline Altitude & 492 \\
\hline Date - number of days & 40097.5864583333 \\
\hline Date & $2009-10-11$ \\
\hline Time & $14: 04: 30$ \\
\hline
\end{tabular}

Figure 3 shows the distribution of points into the map. The data set fed into the modified RBF Network algorithm and pass through the steps showed in figure 2 .

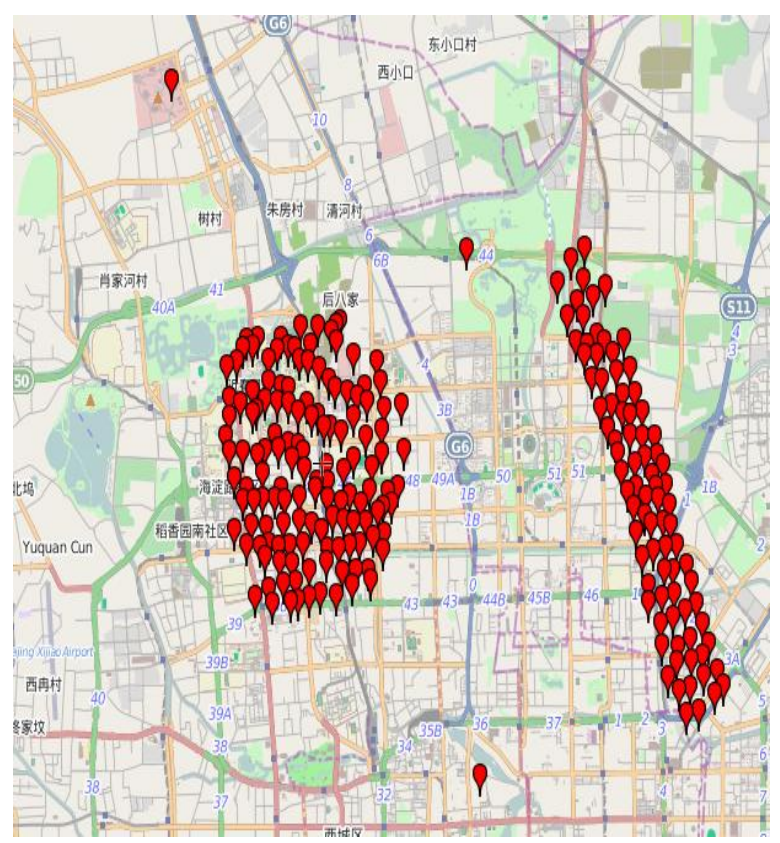

Fig 3: dataset on map 
In the first step, the data is clustered using DBCLUM algorithm which has the ability to detect noise and cluster arbitrary data. Dataset is clustered into two clusters and three points as noise as showed in figure 4 .

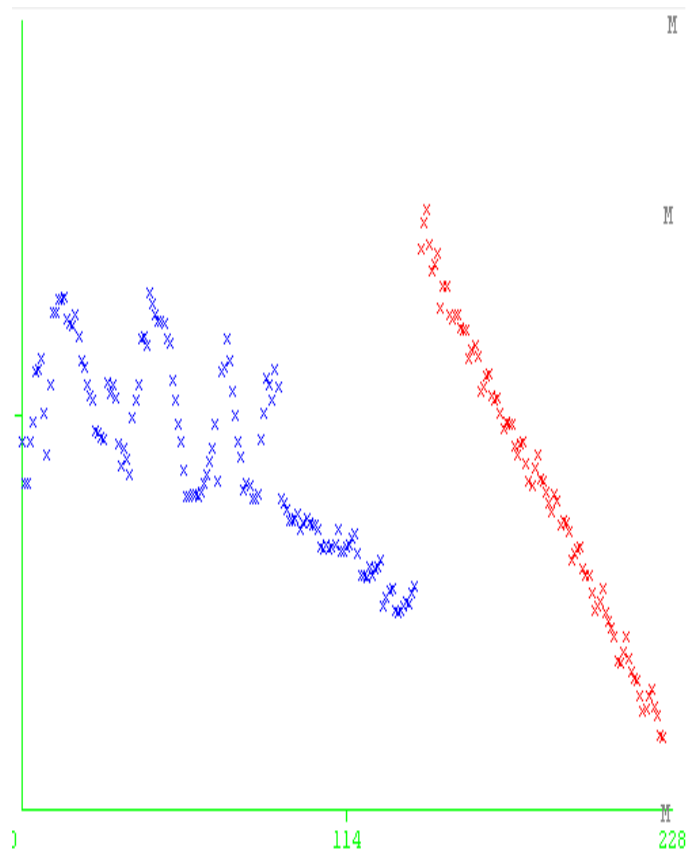

Fig 4: Clustered Dataset

After that, dataset should be labeled to be converted from unsupervised data to supervised data. So go to step two of the algorithm. Labeling data is done by adding a new attribute to the dataset represents class and this by set the value of attribute the cluster name as in table 2 .

Table 2: Labeled Dataset

\begin{tabular}{|c|c|}
\hline Latitude & 39.906631 \\
\hline Longitude & 116.385564 \\
\hline All & 0 \\
\hline Altitude & 492 \\
\hline Date - number of days & 40097.5864583333 \\
\hline Date & $2009-10-11$ \\
\hline Time & $14: 04: 30$ \\
\hline Class & Cluster1 \\
\hline
\end{tabular}

Removing noise from dataset is the following step. Then, compute initial parameters for every cluster. Finally, the prediction step by modified RBF Network which recorded $90 \%$ as a prediction accuracy.

\section{CONCLUSION}

An RBF network using DBCLUM and BP Learning has been modified for mobility prediction. Classifying unsupervised data was an obstacle, proposed is to handle this problem. Firstly, Cluster unsupervised data, prepare it and then the connecting weights were evaluated and finally the whole set of parameter values processed by means of back-propagation. Finally, simulations showed that the method is able to obtain accurate performance.

\section{REFERENCES}

[1] Krumm, J., \& Horvitz, E. (2006). Predestination: Inferring destinations from partial trajectories. In UbiComp 2006: Ubiquitous Computing (pp. 243-260). Springer Berlin Heidelberg.
[2] Liao, L., Patterson, D. J., Fox, D., \& Kautz, H. (2006). Building personal maps from GPS data. Annals of the New York Academy of Sciences, 1093(1), 249-265.

[3] Patterson, D. J., Liao, L., Fox, D., \& Kautz, H. (2003, January). Inferring high-level behavior from low-level sensors. In UbiComp 2003: Ubiquitous Computing (pp. 73-89). Springer Berlin Heidelberg.

[4] Gu, T., Pung, H. K., \& Zhang, D. Q. (2005). A service-oriented middleware for building context-aware services. Journal of Network and computer applications, 28(1), 1-18

[5] Ziebart, B. D., Maas, A. L., Dey, A. K., \& Bagnell, J. A. (2008, September). Navigate like a cabbie: Probabilistic reasoning from observed context-aware behavior. In Proceedings of the 10th international conference on Ubiquitous computing (pp. 322-331). ACM.

[6] Namboodiri, V., \& Gao, L. (2007). Prediction-based routing for vehicular ad hoc networks. Vehicular Technology, IEEE Transactions on, 56(4), 2332-2345.

[7] Su, W., Lee, S. J., \& Gerla, M. (2000). Mobility prediction in wireless networks. In MILCOM 2000. 21st Century Military Communications Conference Proceedings (Vol. 1, pp. 491-495). IEEE.

[8] Liu, G. Y., \& Maguire Jr, G. Q. (1995, November). A predictive mobility management algorithm for wireless mobile computing and communications. In Universal Personal Communications. 1995. Record., 1995 Fourth IEEE International Conference on (pp. 268-272). IEEE.

[9] Samaan, N., \& Karmouch, A. (2005). A mobility prediction architecture based on contextual knowledge and spatial conceptual maps. Mobile Computing, IEEE Transactions on, 4(6), 537-551.

[10] Asahara, A., Maruyama, K., Sato, A., \& Seto, K. (2011, November). Pedestrian-movement prediction based on mixed Markov-chain model. In Proceedings of the 19th ACM SIGSPATIAL International Conference on Advances in Geographic Information Systems (pp. 2533). ACM.

[11] Gambs, S., Killijian, M. O., \& del Prado Cortez, M. N. (2012, April). Next place prediction using mobility markov chains. In Proceedings of the First Workshop on Measurement, Privacy, and Mobility (p. 3). ACM.

[12] Morzy, M. (2007). Mining frequent trajectories of moving objects for location prediction. In Machine Learning and Data Mining in Pattern Recognition (pp. 667-680). Springer Berlin Heidelberg.

[13] Yavaş, G., Katsaros, D., Ulusoy, Ö., \& Manolopoulos, Y. (2005). A data mining approach for location prediction in mobile environments. Data \& Knowledge Engineering, 54(2), 121-146

[14] Capka, J., \& Boutaba, R. (2004). Mobility prediction in wireless networks using neural networks. In Management of Multimedia Networks and Services (pp. 320-333). Springer Berlin Heidelberg.

[15] Kumar, B. V., \& Venkataram, P. (2002). Predictionbased location management using multilayer neural networks. Journal of Indian institute of science, 82(1), 7 22. 
[16] Jeung, H., Liu, Q., Shen, H. T., \& Zhou, X. (2008, April). A hybrid prediction model for moving objects. In Data Engineering, 2008. ICDE 2008. IEEE 24th International Conference on (pp. 70-79). IEEE.

[17] Ying, J. J. C., Lee, W. C., Weng, T. C., \& Tseng, V. S. (2011, November). Semantic trajectory mining for location prediction. In Proceedings of the 19th ACM SIGSPATIAL International Conference on Advances in Geographic Information Systems (pp. 34-43). ACM.

[18] Fawzy, M., Badr, A., Reda, M., \& Farag, I. (2013). DBCLUM: Density-based Clustering and Merging
Algorithm. International Journal of Computer Applications, 79(14), 1-6.

[19] Zheng, Y., Zhang, L., Xie, X., \& Ma, W. Y. (2009, April). Mining interesting locations and travel sequences from GPS trajectories. In Proceedings of the 18th international conference on World wide web (pp. 791800). ACM. 